\title{
dnaJ is a useful phylogenetic marker for alphaproteobacteria
}

Correspondence
Solange Oliveira
ismo@uevora.pt

\section{INTRODUCTION}

The phylum Proteobacteria is composed of five classes, the Alphaproteobacteria, Betaproteobacteria, Gammaproteobacteria, Deltaproteobacteria and Epsilonproteobacteria (Garrity et al., 2005; Stackebrandt et al., 1988). By the end of 2008, more than 380 complete genomes of proteobacteria were available in public databases (http://www. ncbi.nlm.nih.gov/genomes/lproks.cgi). Alphaproteobacteria exhibit an enormous diversity in their morphological and

Abbreviation: HGT, horizontal gene transfer.

The GenBank/EMBL/DDBJ accession numbers for the atpD, dnaJ, gapA, gyrB, recA, rp/B and $16 \mathrm{~S}$ rRNA gene sequences determined in this study are given in bold in Table 1.

Details of PCR primers and conditions, graphical representations of $\mathrm{G}+\mathrm{C}$ content analysis and a dnaJ-based ML tree for Mesorhizobium are available as supplementary material with the online version of this paper. metabolic characteristics and the class is presently recognized solely as a clade in the 16S rRNA gene-based phylogeny (Stackebrandt et al., 1988). Based on 16S rRNA gene trees, the Alphaproteobacteria has been divided into seven orders: Caulobacterales, Rhizobiales, Rhodobacterales, Rhodospirillales, Rickettsiales, Sphingomonadales and Parvularculales (Kersters et al., 2006). The Alphaproteobacteria includes important bacteria that are widely studied, including, for example, the most important genera of soil bacteria able to live in symbiosis with leguminous plants (order Rhizobiales). This interaction between plants and rhizobia leads to the formation of nodules, special structures on plant roots where biological nitrogen fixation takes place, an important process in global nutrient cycling.

Evolutionary relationships among bacteria have been estimated by $16 \mathrm{~S}$ rRNA gene sequence comparisons, since 
this was originally considered to reflect, more or less, organismal phylogeny (Olsen \& Woese, 1993). More recently, it has been shown that rRNA genes may show sequence heterogeneity and undergo horizontal transfer and genetic recombination (Acinas et al., 2004). Furthermore, because of its very high sequence conservation, the 16S rRNA gene has limited usefulness in resolving closely related species. Another disadvantage of this gene is that most bacteria harbour several copies of the $16 \mathrm{~S}$ rRNA gene and, in some cases, different copies have different evolutionary histories. For example, the $16 \mathrm{~S}$ rRNA gene copies of Escherichia coli are divergent (Cilia et al., 1996). For that reason, the search for other genes able to tell an evolutionary story for bacterial species has led to the use of several housekeeping genes, including recA (Eisen, 1995), gyrB (Yamamoto \& Harayama, 1995), rpoD (Yamamoto et al., 2000), atpD (Gaunt et al., 2001) and $g \ln A$ (Turner \& Young, 2000), as alternative phylogenetic markers. The 16S rRNA gene phylogeny is not always in agreement with phylogenies based on housekeeping loci (Xiao et al., 2007). In most cases, 16S rRNA gene and housekeeping gene phylogenies are similar, but phylogenies generated from protein-coding gene sequences frequently show higher resolution and reliability than the corresponding 16S rRNA gene-based phylogeny (Thompson et al., 2004).

Recent phylogenetic studies based on multiple protein sequences from completely sequenced genomes are believed to reveal the 'true' evolutionary history of bacteria. For example, Fukami-Kobayashi et al. (2007) showed a tree of life comprising 167 organisms belonging to the Archaea, Bacteria and Eukarya, based on domain organization of all proteins encoded in each species genome; Ciccarelli et al. (2006) showed a global phylogeny comprising 191 species (Archaea, Bacteria and Eukarya) based on 31 universal proteins. Gupta \& Sneath (2007) studied just proteobacteria, using the amino acid sequences of 10 protein-coding genes. A larger number of proteins (648) was used by Young et al. (2006) to generate a phylogeny that shows the relationship of Rhizobium leguminosarum and its close relatives with completely sequenced genomes. These studies indicate that a large amount of core gene sequence information produces strongly supported and more resolved phylogenies, frequently in disagreement with $16 \mathrm{~S}$ rRNA gene-based phylogenies. Single gene-based phylogenies may now be validated by comparison with presumed organismal phylogeny obtained from multigenic approaches.

Genes coding for molecular chaperones have been used as alternative phylogenetic markers. The most commonly used are groEL (Baldo et al., 2006) and dnaK (Vitorino et al., 2007). Chaperones are known for their role as folding modulators, in sequestering and stabilizing a wide range of polypeptides when the wrong conformational structure is presented (Frydman, 2001). Under standard growth conditions, most of these proteins are expressed constitutively, because they are essential in the folding of nascent polypeptide chains. The bacterial DnaK/J system is a widely studied example of such chaperones.

The DnaJ protein has several domains: the highly conserved J domain (N-terminal), the G/F-rich domain, a conserved region of four repeats with the consensus

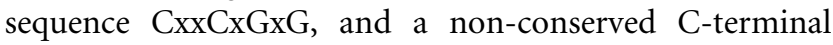
region of variable length (for review see Siegenthaler et al., 2004). In most genomes, dnaK is a single copy gene, often found in the same operon as dnaJ. In Escherichia coli (Gammaproteobacteria), the dnaK operon is bicistronic, comprising the dnaK (1917 bp) and dnaJ (1131 bp) genes (Saito \& Uchida, 1978). The dnaJ gene is sometimes found in more than one copy, as in the high-G+C-content Gram-positive actinobacteria. It has been reported that these different copies may have different physiological functions and distinct evolutionary paths (Ventura et al., 2005).

Its high degree of sequence conservation, functional preservation and universal distribution among bacteria are key features that make the dnaJ gene suitable for inferring phylogenetic relationships. dnaJ has already been used successfully for discrimination of species and subspecies, even within genera in which the $16 \mathrm{~S}$ rRNA gene has insufficient resolution, as in pathogenic bacteria such as Mycobacterium (Morita et al., 2004), Streptococcus (Itoh et al., 2006) and more recently Staphylococcus (Shah et al., 2007) and Vibrio (Nhung et al., 2007). To our knowledge, only one study has reported the use of $d n a J$ as a phylogenetic marker within the Proteobacteria, with the aim of discriminating Legionella pneumophila serogroups (Liu et al., 2003).

Our aim was to find a single core gene with a phylogenetic signal that resembled multilocus phylogenies at higher taxonomic levels (Proteobacteria and Alphaproteobacteria) and with a good phylogenetic signal at a lower taxonomic level, namely for the genus Mesorhizobium. We analysed the phylogenetic signal of the dnaJ gene from 19 proteobacteria of all five classes and then from 20 alphaproteobacteria and compared it with the 16S rRNA gene-based phylogeny, as well as with phylogenies from other studies based on multiple proteins. We also used the dnaJ gene to infer the phylogeny of isolates and type strains within the genus Mesorhizobium (Alphaproteobacteria). The dnaJ-based phylogeny was, in this case, compared to that of five concatenated genes $(a t p D-g a p A-g y r B-r e c A-r p l B)$ and $16 \mathrm{~S}$ rRNA gene-based phylogenies.

\section{METHODS}

Bacterial strains and growth conditions. Rhizobial isolates nodulating chickpea were obtained from two soil samples collected in Portugal using trap plants (Somasegaran \& Hoben, 1994). Five isolates were selected for further study: C-1-Coimbra, C-27bCoimbra, V-15b-Viseu, V-18-Viseu and V-20-Viseu. Four additional isolates obtained previously and identified by $16 \mathrm{~S}$ rRNA gene analysis (Laranjo et al., 2001, 2002, 2004) were used. 
PCR amplifications and sequencing. Bacterial genomes frequently harbour more than one gene annotated as dnaJ. To prevent amplification of multiple copies in the same strain and of different copies in different species, a forward primer in the dnaK gene (see Supplementary Table S1, available in IJSEM Online) was used for PCR amplification (dnaK is a single-copy gene, with very few exceptions), guaranteeing that the target sequence is the dnaJ copy found in the dnaK/J operon. dnaJ amplification by PCR was performed for nine chickpea rhizobia isolates and ten Mesorhizobium type strains. Primers dnaK-F and dnaJ-R were used for PCR amplification. The 16S rRNA gene was amplified by PCR according to Laranjo et al. (2004). The atpD gene, which codes for ATP synthase subunit $\beta$, was amplified using primers atpD-F and atpD-R (Gaunt et al., 2001). The gapA gene, which codes for glyceraldehyde-3-phosphate dehydrogenase, was amplified using primers gap-for and gap-rev. The gyrB gene, which codes for DNA gyrase subunit $\beta$, was amplified using primers gyrBfor_new and gyrBrev_new. The recA gene, which codes for DNA recombinase A, was amplified using primers recA-F and recA-R (Gaunt et al., 2001). The $r p l B$ gene, which codes for the $50 \mathrm{~S}$ ribosomal protein $\mathrm{L} 2$ involved in translation, was amplified using primers L2-for and L2-rev. Primer details are given in Supplementary Table S1.

Total DNA was extracted as described previously (Rivas et al., 2001). All PCR mixtures except that for amplification of the recA gene contained 1 U FideliTaq DNA polymerase (USB), $1 \times$ reaction buffer (with $1.5 \mathrm{mM} \mathrm{MgCl}_{2}$ ), $0.2 \mathrm{mM}$ of each dNTP (Invitrogen), $5 \%$ DMSO (Duchefa), 15-25 pmol of each primer and 7-10 $\mu$ DNA. recA PCRs were prepared with $2 \mathrm{U}$ Taq DNA polymerase (Fermentas), $1 \times$ reaction buffer, $1.5 \mathrm{mM} \mathrm{MgCl}_{2}, 0.2 \mathrm{mM}$ of each dNTP (Invitrogen), $0.004 \%$ BSA (Promega), 25 pmol of each primer and $3 \mu \mathrm{l}$ DNA. Amplification conditions are outlined in Supplementary Table S1.

PCR products were purified using a GFX PCR DNA and gel band purification kit (GE Healthcare) or ExoSAP-It (USB) following the manufacturers' instructions. Sequencing reactions were performed by Macrogen (Korea). For 16S rRNA gene sequencing, two extra primers, IntF and IntR, were used as internal primers for doublestranded sequencing (Laranjo et al., 2004). For dnaJ sequencing, a forward primer located in the $5^{\prime}$-end of the $d n a J$ gene was used (dnaJF; 5'-GCTGGGCGTGCAAAAGGG-3') together with the primer $d n a J-R$. For the remaining genes, the sequencing primers were those used for PCR amplification.

Data analysis. Sequences generated in this study were edited using BioEdit 7.0.5.3 (Hall, 1999) and aligned using CLUSTAL W (Thompson et al., 1994). The sequences were checked manually for correct alignment. Gene sequences from completely sequenced genomes were retrieved from the NCBI complete microbial genomes database.

In order to detect possible events of horizontal gene transfer (HGT) of the dnaJ gene, the $\mathrm{G}+\mathrm{C}$ content and codon usage frequency were analysed for all proteobacteria included in the study (Eisen, 2000). $\mathrm{G}+\mathrm{C}$ content data for the chromosome were retrieved from the NCBI database. $\mathrm{G}+\mathrm{C}$ contents of individual genes were calculated using BioEdit 7.0.5.3 (Hall, 1999). In addition, the $\mathrm{G}+\mathrm{C}$ content of the synonymous third codon position $(\mathrm{G}+\mathrm{C} 3 \mathrm{~s})$ was also calculated for the chromosome and the dnaJ gene using CodonW (Peden, 2000). Codon usage frequency analysis of the $d n a J$ gene was performed with Graphical Codon Usage Analyser 2.0 (http://gcua.schoedl.de/), using the codon usage table of each species available at the Codon Usage Database (http://www.kazusa.or.jp/codon/).

Molecular phylogeny was reconstructed using PHYLIP version 3.67 (Felsenstein, 2006) by the maximum-likelihood (ML) method. Neighbour-joining (NJ) (Saitou \& Nei, 1987) phylogenies were generated using MEGA4 (Tamura et al., 2007). Evolutionary distances were calculated by Kimura's two-parameter model for nucleotide sequence alignments (Kimura, 1980) whereas, for amino acid sequence alignments, the Poisson correction (Nei \& Kumar, 2000) was applied.

For a multilocus approach to the mesorhizobia phylogeny, five protein-coding genes were chosen: atpD, gapA, gyrB, recA and $r p l B$. The incongruence length difference (ILD) test (Bull et al., 1993; Cunningham, 1997) was performed to check whether trees for the different genes were sufficiently similar to allow data combination. The ILD test was performed using PAUP ${ }^{\star} 4.0$ (Swofford, 2003).

\section{RESULTS AND DISCUSSION}

In the phylogenetic studies at higher taxonomic levels, sequences from 19 proteobacteria representing all five classes of the Proteobacteria were used. For further phylogenetic analysis of alphaproteobacteria, 20 strains, from all orders except the Parvularculales, were selected. Finally, at a lower taxonomic level, ten type strains and nine isolates from the genus Mesorhizobium were used. Bacterial strains used in this study are listed in Table 1.

In all proteobacteria used in the present study, the dnaJ gene was found to be encoded adjacent to dnaK, except in epsilonproteobacteria, for which no $d n a K / J$ operon was found among the 11 completely sequenced genomes. In the chromosome of each of the proteobacteria used, only one $d n a J$ gene copy was found that had the expected size and included the four characteristic domains. Other orthologues annotated as dnaJ, often dispersed in the chromosome, were found to lack at least one of the characteristic dnaJ domains. In two species (Ensifer meliloti and Rhizobium etli), a dnaJ homologue was found to be encoded on a plasmid; however, these homologues were partial dnaJ sequences.

Prior to the use of $d n a J$ as a phylogenetic marker, analysis of $\mathrm{G}+\mathrm{C}$ content and codon usage frequency was performed, in order to detect possible HGT events that would compromise the phylogenetic analysis. The $\mathrm{G}+\mathrm{C}$ content of the dnaJ gene was compared with the $\mathrm{G}+\mathrm{C}$ content of the entire chromosome for the alphaproteobacteria (see Supplementary Fig. S1a, available in IJSEM Online) and other proteobacteria used in this study (data not shown). $d n a J \mathrm{G}+\mathrm{C}$ contents are similar to those of the chromosome, while the 16S rRNA gene $\mathrm{G}+\mathrm{C}$ content shows little variation across species (ranging from 50 to $57 \mathrm{~mol} \%$ ), regardless of the $\mathrm{G}+\mathrm{C}$ content of the chromosome (ranging from 29 to $69 \mathrm{~mol} \%$ ). For example, in Rickettsia prowazekii, the 16S rRNA gene $\mathrm{G}+\mathrm{C}$ content $(51 \mathrm{~mol} \%)$ is clearly higher than that of the chromosome $(29 \mathrm{~mol} \%)$, whereas the $\mathrm{G}+\mathrm{C}$ content of the dnaJ gene $(36 \mathrm{~mol} \%)$ is closer to the chromosomal value. Core genes, such as recA or $d n a K$, present a $\mathrm{G}+\mathrm{C}$ content similar to that of the whole chromosome. No evidence of HGT events was provided by this analysis for the alphaproteobacteria or the other proteobacteria used (data not shown). Nevertheless, the possibility of dnaJ HGT occurring between organisms with similar $\mathrm{G}+\mathrm{C}$ contents cannot be excluded. 
Table 1. Strains of the Proteobacteria with corresponding accession numbers or gene IDs of sequences used in this study

Accession numbers for sequences resulting from this study are shown in bold.

\begin{tabular}{|c|c|c|c|c|c|c|c|c|c|}
\hline Strain & Genome & 16S rRNA gene & $d n a J$ & dnaK & $\operatorname{atpD}$ & gapA & gyrB & $\operatorname{rec} A$ & $r p l B$ \\
\hline \multicolumn{10}{|l|}{ Alphaproteobacteria } \\
\hline Agrobacterium tumefaciens C58 & NC_003062 & 3244993 & 1137637 & 1137638 & & & & & \\
\hline Bradyrhizobium japonicum USDA 110 & NC_004463 & 1055154 & 1049300 & 1049096 & 1054445 & 1055273 & 1047796 & 1048547 & 1051246 \\
\hline Brucella suis $1330^{\mathrm{T}}$ & NC_004310 & 1167560 & 1167829 & 1167828 & & & & & \\
\hline Caulobacter crescentus CB15 & NC_002696 & 943247 & 944076 & 944075 & & & & & \\
\hline Ensifer meliloti 1021 & NC_003047 & 1234653 & 1231814 & 1234653 & 1234718 & 1234439 & 1231637 & 1233464 & 1233011 \\
\hline Erythrobacter litoralis HTCC2594 & NC_007722 & 3868981 & 3870763 & 3870762 & & & & & \\
\hline Gluconobacter oxydans $621 \mathrm{H}$ & NC_006677 & 3248602 & 3250315 & 3250314 & & & & & \\
\hline Granulibacter bethesdensis $\mathrm{CGDNIH}^{\mathrm{T}}$ & NC_008343 & 4276889 & 4274426 & 4274427 & & & & & \\
\hline Maricaulis maris MCS10 & NC_008347 & 4285688 & 4283983 & 4283981 & & & & & \\
\hline \multicolumn{10}{|l|}{ Genus Mesorhizobium } \\
\hline M. amorphae ACCC $19665^{\mathrm{T}}$ & & AF041442 & EF504296 & & AY493453 & AM072544 & AM076341 & AY494816 & AM076350 \\
\hline M. chacoense LMG $19008^{\mathrm{T}}$ & & AJ278249 & EU273806 & & AY493460 & AM072546 & AM076343 & AY494825 & AM076352 \\
\hline M. ciceri $\mathrm{UPM}-\mathrm{Ca} 7^{\mathrm{T}}$ & & DQ444456 & EF504297 & & AJ294395 & AM072545 & AM076342 & AJ294367 & AM076351 \\
\hline M. ciceri $27-$-Beja & & & EF504304 & & & & & & \\
\hline M. huakuii CCBAU $2609^{\mathrm{T}}$ & & D12797 & EF504298 & & AJ294394 & AM072547 & AM076344 & AJ294370 & AM076353 \\
\hline M. loti $\mathrm{LMG} 6125^{\mathrm{T}}$ & & X67229 & EU053202 & & EU039868 & EU273807 & EU273810 & EU039875 & EU273813 \\
\hline M. loti MAFF 303099 & NC_002678 & 3205748 & 1228214 & 1228215 & & & & & \\
\hline M. loti 75-Elvas & & & EF504306 & & & & & & \\
\hline M. mediterraneum UPM-Ca $36^{\mathrm{T}}$ & & L38825 & EF504299 & & AM418768 & AM072549 & AM076346 & AJ294369 & AM076355 \\
\hline M. mediterraneum 29-Beja & & & EF504305 & & & & & & \\
\hline M. plurifarium ORS $1032^{\mathrm{T}}$ & & Y14158 & EF504300 & & AM076366 & AM072550 & AM076347 & AY494824 & AM076356 \\
\hline M. septentrionale HAMBI $2582^{\mathrm{T}}$ & & AF508207 & EF504301 & & DQ659498 & EU273808 & EU273811 & DQ444304 & EU273814 \\
\hline M. temperatum HAMBI $2583^{\mathrm{T}}$ & & AF508208 & EF504302 & & DQ659499 & EU273809 & EU273812 & DQ444305 & EU273815 \\
\hline M. tianshanense $\mathrm{A}-1 \mathrm{BS}^{\mathrm{T}}$ & & AF041447 & EF504303 & & AM076367 & AM072551 & AM076348 & AJ294368 & AM076357 \\
\hline M. tianshanense 93-Évora & & & EF504307 & & & & & & \\
\hline Mesorhizobium sp. C-1-Coimbra & & & EF504308 & & & & & & \\
\hline Mesorhizobium sp. C-27b-Coimbra & & & EF504309 & & & & & & \\
\hline Mesorhizobium sp. V-15b-Viseu & & & EF504310 & & & & & & \\
\hline Mesorhizobium sp. V-18-Viseu & & & EF504311 & & & & & & \\
\hline Mesorhizobium sp. V-20-Viseu & & & EF504312 & & & & & & \\
\hline Nitrobacter winogradskyi Nb- $255^{\mathrm{T}}$ & NC_007406 & 3676957 & 3676654 & 3676653 & & & & & \\
\hline Novosphingobium aromaticivorans DSM $12444^{\mathrm{T}}$ & NC_007794 & 3917048 & 3917700 & 3917701 & & & & & \\
\hline Rhizobium leguminosarum bv. viciae 3841 & NC_008380 & 4403801 & 4402721 & 4402722 & & & & & \\
\hline Rhizobium etli $\mathrm{CFN} 42^{\mathrm{T}}$ & NC_007761 & 3893174 & 3890921 & 3890922 & AJ294404 & 3891389 & 3892150 & AJ294375 & 3892587 \\
\hline Rhodobacter sphaeroides $2.4 .1^{\mathrm{T}}$ & NC_007493 & 3718805 & 3718165 & 3718166 & & & & & \\
\hline Rhodopseudomonas palustris CGA009 & NC_005296 & 2690886 & 2691660 & 2689800 & & & & & \\
\hline Rhodospirillum rubrum ATCC $11170^{\mathrm{T}}$ & NC_007643 & 3833695 & 3835440 & 3837010 & & & & & \\
\hline Rickettsia prowazekii Madrid E & NC_000963 & 883924 & 883882 & 883879 & & & & & \\
\hline
\end{tabular}


Table 1. cont.

\begin{tabular}{|c|c|c|c|c|c|c|c|c|c|}
\hline Strain & Genome & $16 S$ rRNA gene & $d n a J$ & dnaK & $a t p D$ & gapA & gyrB & recA & $r p l B$ \\
\hline Roseobacter denitrificans Och $114^{\mathrm{T}}$ & NC_008209 & 4198019 & 4198416 & 4198415 & & & & & \\
\hline Silicibacter pomeroyi DSS $-3^{\mathrm{T}}$ & NC_003911 & 3196347 & 3196511 & 3195579 & & & & & \\
\hline \multicolumn{10}{|l|}{ Betaproteobacteria } \\
\hline Azoarcus sp. EbN1 & NC_006513 & 3180819 & 3179956 & & & & & & \\
\hline Bordetella pertussis Tohama I & NC_002929 & 3131280 & 2666530 & & & & & & \\
\hline Burkholderia ambifaria $\mathrm{AMMD}^{\mathrm{T}}$ & NC_008390 & 4308934 & 4309294 & & & & & & \\
\hline Nitrosospira multiformis ATCC $25196^{\mathrm{T}}$ & NC_007614 & 3784866 & 3784939 & & & & & & \\
\hline Ralstonia solanacearum GMI1000 & NC_003295 & 1220256 & 1221481 & & & & & & \\
\hline \multicolumn{10}{|l|}{ Gammaproteobacteria } \\
\hline Aeromonas hydrophila subsp. hydrophila ATCC $7966^{\mathrm{T}}$ & NC_008570 & 4488614 & 4488918 & & & & & & \\
\hline Escherichia coli $\mathrm{K}-12 \mathrm{MG} 1655$ & NC_000913 & 948332 & 944753 & & & & & & \\
\hline Legionella pneumophila Paris & NC_006368 & 3116667 & 3118141 & & & & & & \\
\hline Pseudomonas aeruginosa $\mathrm{PAO} 1$ & NC_002516 & 3240252 & 881760 & & & & & & \\
\hline Vibrio cholerae O1 bv. eltor N16961 & NC_002505 & 2614447 & 2614523 & & & & & & \\
\hline \multicolumn{10}{|l|}{ Deltaproteobacteria } \\
\hline Geobacter sulfurreducens $\mathrm{PCA}^{\mathrm{T}}$ & NC_002939 & 2685615 & 2688568 & & & & & & \\
\hline Pelobacter carbinolicus DSM $2380^{\mathrm{T}}$ & NC_007498 & 3725044 & 3722901 & & & & & & \\
\hline \multicolumn{10}{|l|}{ Epsilonproteobacteria } \\
\hline Campylobacter jejuni RM1221 & NC_003912 & 3232519 & 3231902 & & & & & & \\
\hline Helicobacter pylori 26695 & NC_000915 & 899682 & 898772 & & & & & & \\
\hline
\end{tabular}


In order to detect dnaJ recently acquired by HGT, the $\mathrm{G}+\mathrm{C} 3$ s content was also analysed (Sharp et al., 2005). The $\mathrm{G}+\mathrm{C} 3 \mathrm{~s}$ of the chromosome shows the average $\mathrm{G}+\mathrm{C} 3 \mathrm{~s}$ of the chromosomal protein-coding genes. Highly expressed genes display higher codon biases than the average, as is the case for genes coding for some chaperones, such as dnaK (Karlin et al., 2004). A gene with a G+C3s content significantly different from the average of the chromosome and from that of highly expressed genes is probably a recently acquired gene (Karlin et al., 2004). In the alphaproteobacteria (Supplementary Fig. S1b), the dnaJ gene has, in most cases, a G $+\mathrm{C} 3$ s content between that of the chromosome and that of $d n a K$ (a highly expressed gene). The only two species that show a $\mathrm{G}+\mathrm{C} 3 \mathrm{~s}$ for $d n a J$ clearly below the average of the chromosome genes are Agrobacterium tumefaciens and Roseobacter denitrificans. Therefore, dnaJ from these two species could have been acquired by HGT. For the remaining proteobacteria (data not shown), the $\mathrm{G}+\mathrm{C} 3 \mathrm{~s}$ values for $d n a J$ are often very similar to those of the corresponding chromosome and, in some cases, similar to the value for $d n a K$ (for example, in Burkholderia ambifaria), so no evidence was found for HGT of the dnaJ gene.

Another approach to look for evidence of HGT is to compare the codon usage table of each organism with the codon usage frequency of a given gene. If the dnaJ gene includes a large number of codons that are uncommonly used by the bacterium, it is likely that this gene was acquired by HGT. Codon usage analysis did not reveal any discrepancies that could clearly indicate HGT events (data not shown). Nonetheless, the dnaJ gene of Agrobacterium tumefaciens shows the highest percentage of uncommonly used codons. This discrepancy in codon usage together with the $\mathrm{G}+\mathrm{C} 3$ s result raises doubts about the origin of the dnaJ gene in Agrobacterium tumefaciens, suggesting that it might have been acquired by HGT. Thus, the position of Agrobacterium tumefaciens in the dnaJ-based phylogeny should be regarded with caution.

In general, our analysis of $\mathrm{G}+\mathrm{C}$ and $\mathrm{G}+\mathrm{C} 3$ s contents suggests that dnaJ is a core gene and is not commonly subject to HGT between species. These features are important in a good phylogenetic marker, so we used it for phylogenetic analysis of members of the Proteobacteria, Alphaproteobacteria and Mesorhizobium.

Phylogenetic analyses were performed using both the ML and NJ methods. In general, the two methods generated trees with the same topology.

\section{The Proteobacteria}

The Proteobacteria were represented in this study by 19 strains with completely sequenced genomes. The topology of the proteobacterial maximum-likelihood tree obtained with DnaJ amino acid sequences (Fig. 1a) is identical to that based on $16 \mathrm{~S}$ rRNA gene sequences (Fig. 1b). The five classes of the Proteobacteria are well defined and their branching order is the same in both trees: the Betaproteobacteria is closer to the Gammaproteobacteria, and these two classes group first with the Alphaproteobacteria, then with the Deltaproteobacteria and finally with the Epsilonproteobacteria.

The branching order of all classes of the Proteobacteria, in both DnaJ and 16S rRNA gene phylogenies (Fig. 1), is in agreement with several recent studies that combine data from a large number of protein-coding genes, although
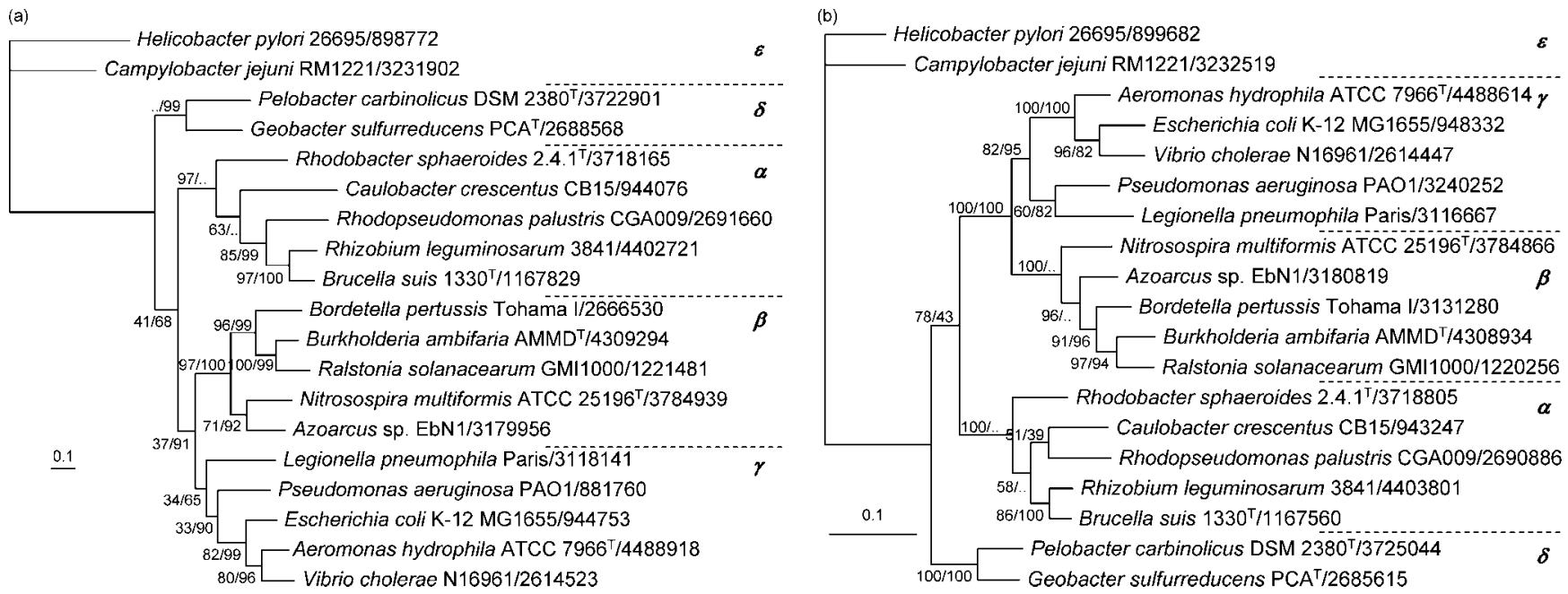

Fig. 1. Phylogeny of 19 members of the Proteobacteria from all five classes (indicated by Greek letters) based on analysis of the DnaJ amino acid sequence (a) and the 16S rRNA gene sequence (b). Trees were generated by ML. The first bootstrap percentage indicated on internal branches corresponds to the ML method (100 replicates) and the second to the NJ method (1000 replicates); dots indicate that nodes were not resolved using that method. Bars, 0.1 substitutions per site (ML). 
some different species were used (Ciccarelli et al., 2006; Fukami-Kobayashi et al., 2007; Gupta \& Sneath, 2007). It is noteworthy that some of these broader phylogenies showed that the Deltaproteobacteria and Epsilonproteobacteria may form one group, apart from the remaining classes of the Proteobacteria (Ciccarelli et al., 2006; Fukami-Kobayashi et al., 2007; Gupta, 2000).

Although the same branching order of classes of the Proteobacteria is generated in both phylogenies, the relationships between species within each cluster are different in a few cases. For example, relationships among the gammaproteobacteria Aeromonas hydrophila, Vibrio cholerae and Escherichia coli in the 16S rRNA gene-based tree are consistent with the phylogeny shown in a previous study using 31 concatenated protein sequences and comprising a large group of gammaproteobacteria (Seshadri et al., 2006), but the DnaJ tree shows a different relationship.

Despite such minor discrepancies, the global congruence found between the 16S rRNA gene and the DnaJ phylogeny and the agreement with other phylogenies based on multilocus data indicate a good performance of $d n a J$ in reconstructing proteobacteria phylogeny.

\section{The Alphaproteobacteria}

To study the phylogenetic signal of the dnaJ gene at a lower taxonomic level, 20 strains belonging to the Alphaproteobacteria with completely sequenced genomes were used. In contrast to the $16 \mathrm{~S}$ rRNA gene phylogeny (Fig. 2b), the phylogeny based on DnaJ sequences (Fig. 2a) is in agreement with the currently accepted relationships among genera and species within this group of bacteria obtained in other studies based on large amounts of sequence information (Ciccarelli et al., 2006; Gupta, 2005; Gupta \& Sneath, 2007).

Both the DnaJ and 16S rRNA gene phylogenies (Fig. 2) show Rhodospirillum rubrum, Gluconobacter oxydans and Granulibacter bethesdensis (cluster A) and Rickettsia prowazekii in a distant position from the other clusters (the letters denote the clusters generated in the DnaJ phylogeny). However, clusters B, C, D and E are differently related in the two phylogenies. In the DnaJ phylogeny, cluster B (Agrobacterium tumefaciens, Brucella suis, Ensifer meliloti, Mesorhizobium loti, Rhizobium etli and Rhizobium leguminosarum) is closely related to cluster C (Bradyrhizobium japonicum, Nitrobacter winogradskyi and Rhodopseudomonas palustris) and then these clusters group first with cluster D (Caulobacter crescentus, Erythrobacter litoralis, Maricaulis maris and Novosphingobium aromaticivorans) and finally with cluster E (Rhodobacter sphaeroides, Roseobacter denitrificans and Silicibacter pomeroyi). In contrast to the DnaJ-based phylogeny, the 16S rRNA gene phylogeny shows cluster B closer to cluster E, then these two clusters group with two species of cluster D and finally with cluster C. Furthermore, in the $16 \mathrm{~S}$ rRNA gene phylogeny, cluster D from the DnaJ phylogeny is dissolved and Maricaulis maris groups with cluster E while Caulobacter crescentus groups with cluster C species.

The relationships among different species inferred from DnaJ sequence comparisons are consistent with those inferred in previous studies, namely the phylogeny based on concatenated sequences of 10 proteins (Gupta \& Sneath, 2007) and the tree of life generated from the concatenated alignment of 31 universal protein families (Ciccarelli et al., 2006). In the DnaJ-based phylogeny, all members of the Rhizobiales cluster together (branch $\mathrm{B}+\mathrm{C}$ ), thus Bradyrhizobium japonicum (cluster C) is closer to

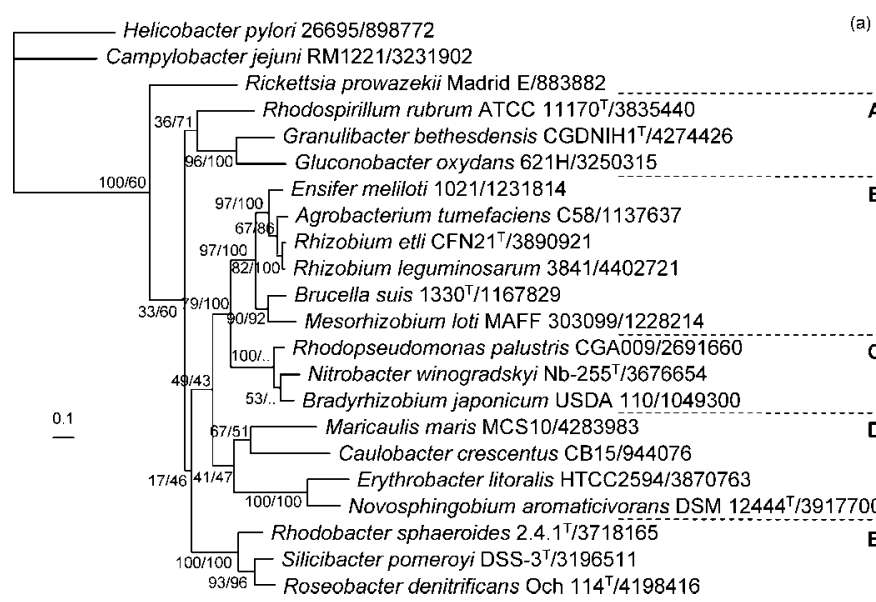

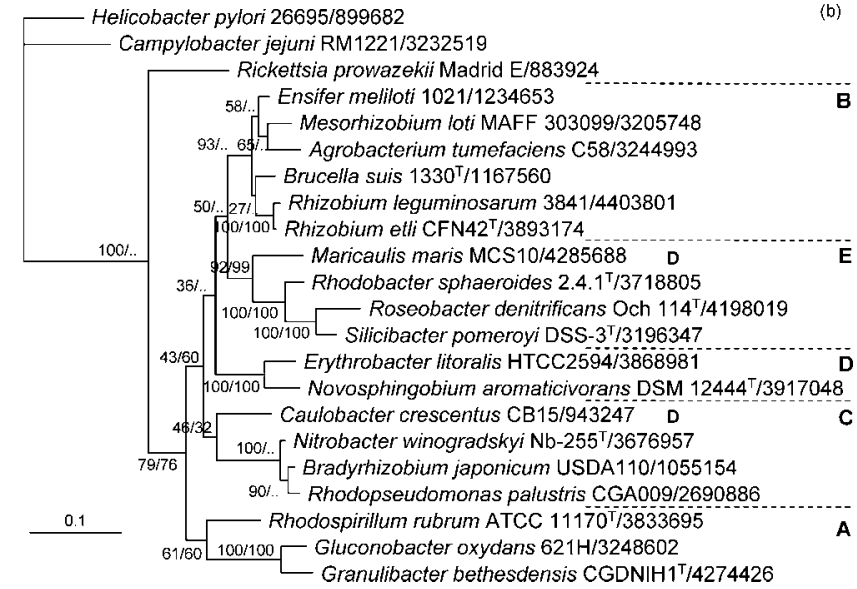

(b)

\section{(1)} 穴 C A Granulibacter bethesdensis CGDNIH1 $1^{\top} / 4274426$

Fig. 2. Phylogeny of 20 members of the Alphaproteobacteria, based on analysis of the DnaJ amino acid sequence (a) and the 16S rRNA gene sequence (b). Trees were generated by ML. Two species of the Epsilonproteobacteria were used as an outgroup. The first bootstrap percentage indicated on internal branches corresponds to the ML method (100 replicates) and the second to the NJ method (1000 replicates); dots indicate that nodes were not resolved using that method. Bar, 0.1 substitutions per site (ML). Letters denote clusters generated in the DnaJ phylogeny. 
Mesorhizobium loti (cluster B) than to Caulobacter crescentus (Caulobacterales in cluster D), as reported before (Ciccarelli et al., 2006; Gupta \& Sneath, 2007). In contrast, the 16S rRNA gene-based phylogeny shows Bradyrhizobium japonicum (cluster C) close to Caulobacter crescentus (cluster D) and distant from Mesorhizobium loti (cluster B). Moreover, within cluster B, the DnaJ-based phylogeny shows Ensifer meliloti closer to Agrobacterium tumefaciens and Mesorhizobium loti closer to Brucella suis, which is in full agreement with the phylogeny proposed by Young et al. (2006) based on the concatenated sequences of 638 proteins (the present study uses Brucella suis rather than Brucella melitensis) and with the multilocus tree of life presented by Ciccarelli et al. (2006), and not concordant with the 16S rRNA gene phylogeny. Therefore, the possible HGT origin of dnaJ from Agrobacterium tumefaciens (cluster B) suggested by the $\mathrm{G}+\mathrm{C}$ content analysis was not confirmed.

The coherence between our results using dnaJ and those from broader phylogenies using multiple protein-coding genes (Ciccarelli et al., 2006; Gupta \& Sneath, 2007) shows that $d n a J$ has a better phylogenetic signal than the $16 \mathrm{~S}$ rRNA gene in reconstructing the phylogeny of alphaproteobacteria.

\section{The genus Mesorhizobium}

The high level of sequence conservation of the 16S rRNA gene represents a limitation on the use of this gene for closely related bacteria. In the genus Mesorhizobium, other genes have been used for phylogenetic purposes, such as dnaK (Stepkowski et al., 2003), atpD and recA (Vinuesa et al., 2005). The non-coding 16S-23S rRNA intergenic spacer (ITS) has also been used (Rivas et al., 2007). Relationships between mesorhizobial species are different depending on the gene used. Due to this unclear positioning and to the lack of a multilocus analysis focusing on mesorhizobia, there is no generally accepted phylogeny of this genus.

In order to obtain a reliable phylogenetic tree for the genus Mesorhizobium that could be compared to the dnaJ- and $16 \mathrm{~S}$ rRNA gene-based phylogenies, five core genes (atpD, gapA, gyrB, recA and $r p l B$ ) from ten mesorhizobia type strains were partially sequenced. The ILD test was applied to find out which genes could be combined, and the results allow the concatenation of the amino acid sequences of all genes. The ML tree based on the concatenated alignment of the five genes (approx. 770 amino acids long), shown in Fig. 3(a), reveals the putative organismal phylogeny among Mesorhizobium species. Both the DnaJ tree (Fig. 3b) and the 16S rRNA gene tree (Fig. 3c) were compared with the hypothetical organismal phylogeny to evaluate the accuracy of the phylogenetic signal of each single gene.

The 16S rRNA gene tree shows a different topology from the multilocus tree. According to the multilocus analysis, Mesorhizobium huakuii groups with Mesorhizobium ciceri
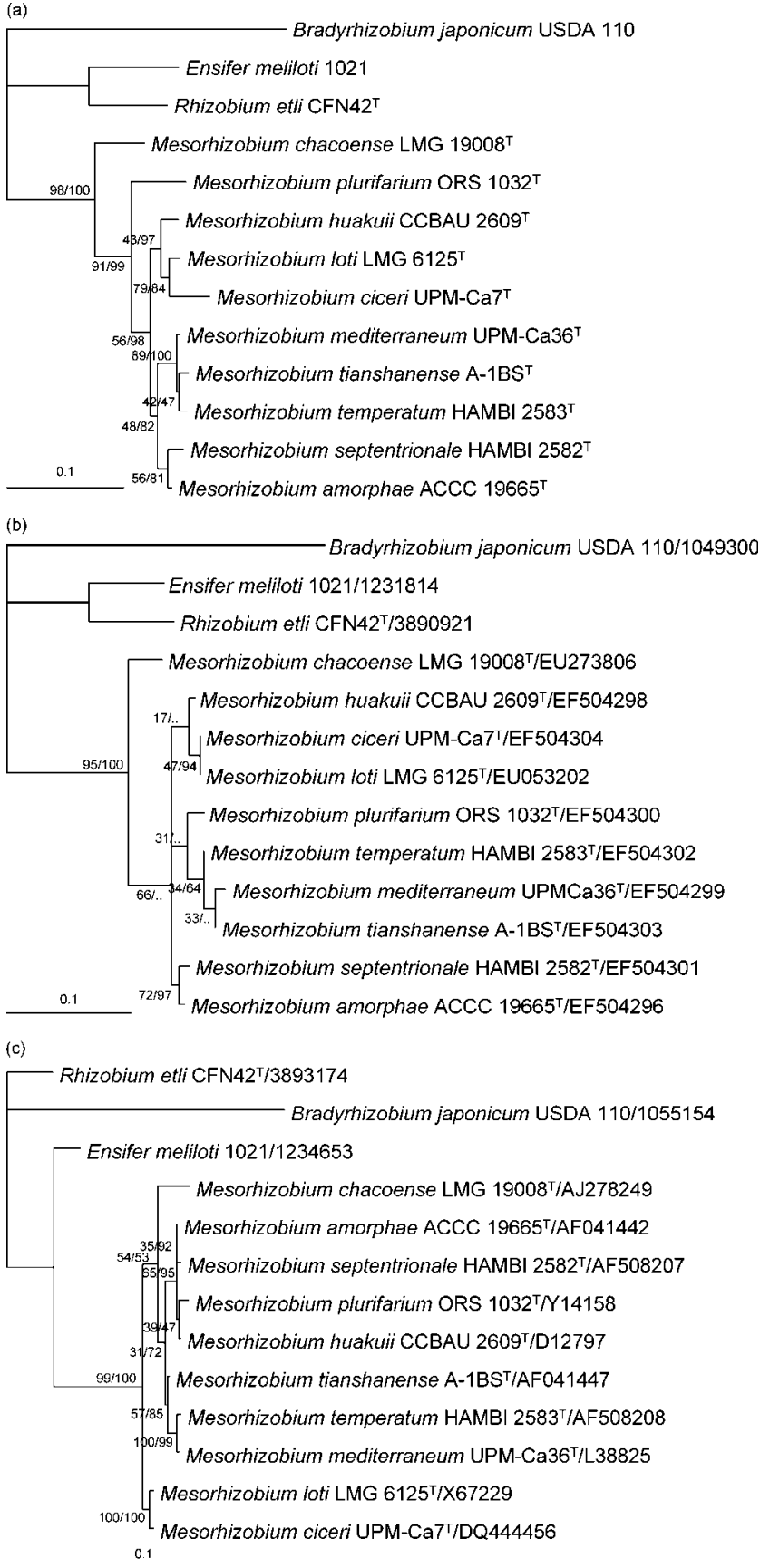

Fig. 3. Phylogeny based on the concatenated amino acid sequence alignment of AtpD-GapA-GyrB-RecA-RplB (a), the DnaJ sequence (b) and the 16S rRNA gene sequence (c) for the genus Mesorhizobium. Trees were generated by ML. The first bootstrap percentage indicated on internal branches corresponds to the ML method (100 replicates) and the second to the NJ method (1000 replicates); dots indicate that nodes were not resolved using that method. Bars, 0.1 substitutions per site (ML).

and Mesorhizobium loti in the deeper branching of the dendrogram. In contrast to this, the 16S rRNA gene-based phylogeny shows Mesorhizobium ciceri and Mesorhizobium 
loti apart from the remaining eight mesorhizobia type strains. The concatenated tree shows Mesorhizobium chacoense and Mesorhizobium plurifarium as the most divergent species. The only two groups generated in both trees are Mesorhizobium mediterraneum/Mesorhizobium temperatum/Mesorhizobium tianshanense and Mesorhizobium amorphae/Mesorhizobium septentrionale. Among the single-gene trees, the DnaJ (Fig. 3b) and GyrB (data not shown) trees show the topologies most consistent with that of the multilocus analysis, although they have some low bootstrap values. The similarity between the DnaJ-based tree and the putative Mesorhizobium phylogeny derived from the multilocus analysis suggests that this gene is not commonly subject to HGT between Mesorhizobium species.

The present multilocus phylogenetic analysis may contribute to the clarification of the phylogenetic relationships among Mesorhizobium species, namely the proximity of Mesorhizobium ciceri, Mesorhizobium loti and Mesorhizobium huakuii. Interestingly, analysis of phenotypic data from a large set of chickpea mesorhizobia isolates also supported a closer relationship between Mesorhizobium loti/Mesorhizobium ciceri and Mesorhizobium huakuii isolates (Alexandre et al., 2006).

In order to evaluate the suitability of $d n a J$ sequences for identifying native chickpea rhizobia isolates, a dnaJ-based tree including nine isolates was generated (Supplementary Fig. S2). At this taxonomic level, nucleotide sequences were used, since the amino acid sequence-based tree showed low resolution and low bootstrap support (data not shown). Although the relative positioning of some type strains was different from the concatenated tree (Fig. 3a) and the $16 \mathrm{~S}$ rRNA gene-based tree (Fig. 3c), identification of isolates based on dnaJ sequences is consistent with identification based on the 16S rRNA gene (Laranjo et al., 2004). The present phylogenetic analysis of native rhizobia reveals a high diversity of species able to nodulate chickpea, namely isolates close to Mesorhizobium ciceri, Mesorhizobium huakuii, Mesorhizobium loti, Mesorhizobium mediterraneum, Mesorhizobium temperatum and Mesorhizobium tianshanense, confirming previous analyses of chickpea mesorhizobia (Laranjo et al., 2004; Rivas et al., 2007).

For decades, the 16S rRNA gene has been the most ubiquitous gene used for accessing bacterial phylogeny, but this has been changing. Many other core genes are now used as phylogenetic markers in order to infer species phylogeny and evolution. Bacterial dnaJ is a core gene, as confirmed by its chromosomal location and a $\mathrm{G}+\mathrm{C}$ content similar to that of the chromosome. This gene is present as a single 'true' copy, usually in the same operon as $d n a K$, which allows its specific amplification by PCR. No evidence that $d n a J$ had been subject to a HGT event was found in the proteobacteria that were examined, and the phylogenetic signal of this gene was consistent with studies based on multiple protein sequences. In contrast to $16 \mathrm{~S}$ rRNA gene trees, the alphaproteobacterial DnaJ phylogenetic tree showed a global topology that resembled the presumed organismal phylogeny inferred from multiple protein sequences from complete genomes. For these reasons, we suggest that a single core gene, such as dnaJ, can be used as a phylogenetic marker for proteobacteria at the level of phylum (Proteobacteria) and class (Alphaproteobacteria). At the level of the genus Mesorhizobium, the dnaJ gene can be used for identification of isolates. This study highlights the usefulness of the dnaJ gene as a single alternative phylogenetic marker for alphaproteobacteria.

\section{ACKNOWLEDGEMENTS}

This work was supported by the Fundação para a Ciência e Tecnologia (FCT) (POCTI/BME/44140/2002) and co-financed by EU-FEDER. A. A. acknowledges a PhD fellowship (SFRH/BD/18162/ 2004) and M.L. acknowledges a post-doctoral fellowship (SFRH/ BPD/27008/2006) from FCT. The contribution of J.P.W.Y. was funded by the Natural Environment Research Council, UK.

\section{REFERENCES}

Acinas, S. G., Marcelino, L. A., Klepac-Ceraj, V. \& Polz, M. F. (2004). Divergence and redundancy of $16 \mathrm{~S}$ rRNA sequences in genomes with multiple rrn operons. J Bacteriol 186, 2629-2635.

Alexandre, A., Laranjo, M. \& Oliveira, S. (2006). Natural populations of chickpea rhizobia evaluated by antibiotic resistance profiles and molecular methods. Microb Ecol 51, 128-136.

Baldo, L., Bordenstein, S., Wernegreen, J. J. \& Werren, J. H. (2006). Widespread recombination throughout Wolbachia genomes. Mol Biol Evol 23, 437-449.

Bull, J. J., Huelsenbeck, J. P., Cunningham, C. W., Swofford, D. L. \& Waddell, P. J. (1993). Partitioning and combining data in phylogenetic analysis. Syst Biol 42, 384-397.

Ciccarelli, F. D., Doerks, T., von Mering, C., Creevey, C. J., Snel, B. \& Bork, P. (2006). Toward automatic reconstruction of a highly resolved tree of life. Science 311, 1283-1287.

Cilia, V., Lafay, B. \& Christen, R. (1996). Sequence heterogeneities among $16 \mathrm{~S}$ ribosomal RNA sequences, and their effect on phylogenetic analyses at the species level. Mol Biol Evol 13, 451-461.

Cunningham, C. W. (1997). Can three incongruence tests predict when data should be combined? Mol Biol Evol 14, 733-740.

Eisen, J. A. (1995). The RecA protein as a model molecule for molecular systematic studies of bacteria: comparison of trees of RecAs and $16 \mathrm{~S}$ rRNAs from the same species. J Mol Evol 41, 1105-1123.

Eisen, J. A. (2000). Horizontal gene transfer among microbial genomes: new insights from complete genome analysis. Curr Opin Genet Dev 10, 606-611.

Felsenstein, J. (2006). PHYLIP (phylogeny inference package). Distributed by the author. Department of Genome Sciences, University of Washington, Seattle, USA.

Frydman, J. (2001). Folding of newly translated proteins in vivo: the role of molecular chaperones. Annu Rev Biochem 70, 603-647.

Fukami-Kobayashi, K., Minezaki, Y., Tateno, Y. \& Nishikawa, K. (2007). A tree of life based on protein domain organizations. Mol Biol Evol 24, 1181-1189.

Garrity, G. M., Bell, J. A. \& Lilburn, T. (2005). Phylum XIV. Proteobacteria phyl. nov. In Bergey's Manual of Systematic 
Bacteriology, 2nd edn, vol. 2, part B, p. 1. Edited by D. J. Brenner, N. R. Krieg, J. T. Staley \& G. M. Garrity. New York: Springer.

Gaunt, M. W., Turner, S. L., Rigottier-Gois, L., Lloyd-Macgilp, S. A. \& Young, J. P. W. (2001). Phylogenies of atpD and recA support the small subunit rRNA-based classification of rhizobia. Int J Syst Evol Microbiol 51, 2037-2048.

Gupta, R. S. (2000). The phylogeny of proteobacteria: relationships to other eubacterial phyla and eukaryotes. FEMS Microbiol Rev 24, 367402.

Gupta, R. S. (2005). Protein signatures distinctive of alpha proteobacteria and its subgroups and a model for alpha-proteobacterial evolution. Crit Rev Microbiol 31, 101-135.

Gupta, R. S. \& Sneath, P. H. A. (2007). Application of the character compatibility approach to generalized molecular sequence data: branching order of the proteobacterial subdivisions. J Mol Evol 64, 90-100.

Hall, T. A. (1999). BioEdit: a user-friendly biological sequence alignment editor and analysis program for Windows 95/98/NT. Nucleic Acids Symp Ser 41, 95-98.

Itoh, Y., Kawamura, Y., Kasai, H., Shah, M. M., Nhung, P. H., Yamada, M., Sun, X., Koyana, T., Hayashi, M. \& other authors (2006). dnaJ and gyrB gene sequence relationship among species and strains of genus Streptococcus. Syst Appl Microbiol 29, 368-374.

Karlin, S., Theriot, J. \& Mrazek, J. (2004). Comparative analysis of gene expression among low $\mathrm{G}+\mathrm{C}$ gram-positive genomes. Proc Natl Acad Sci U S A 101, 6182-6187.

Kersters, K., De Vos, P., Gillis, M., Swings, J., Vandamme, P. \& Stackebrandt, E. (2006). Introduction to the Proteobacteria. In The Prokaryotes: a Handbook on the Biology of Bacteria, 3rd edn, vol. 5, pp. 3-37. Edited by M. Dworkin, S. Falkow, E. Rosenberg, K. H. Schleifer and E. Stackebrandt. New York: Springer.

Kimura, M. (1980). A simple method for estimating evolutionary rates of base substitutions through comparative studies of nucleotide sequences. J Mol Evol 16, 111-120.

Laranjo, M., Rodrigues, R., Alho, L. \& Oliveira, S. (2001). Rhizobia of chickpea from southern Portugal: symbiotic efficiency and genetic diversity. J Appl Microbiol 90, 662-667.

Laranjo, M., Branco, C., Soares, R., Alho, L., Carvalho, M. \& Oliveira, S. (2002). Comparison of chickpea rhizobia isolates from diverse Portuguese natural populations based on symbiotic effectiveness and DNA fingerprint. J Appl Microbiol 92, 1043-1050.

Laranjo, M., Machado, J., Young, J. P. W. \& Oliveira, S. (2004). High diversity of chickpea Mesorhizobium species isolated in a Portuguese agricultural region. FEMS Microbiol Ecol 48, 101-107.

Liu, H., Li, Y., Huang, X., Kawamura, Y. \& Ezaki, T. (2003). Use of the dnaJ gene for the detection and identification of all Legionella pneumophila serogroups and description of the primers used to detect $16 \mathrm{~S}$ rDNA gene sequences of major members of the genus Legionella. Microbiol Immunol 47, 859-869.

Morita, Y., Maruyama, S., Kabeya, H., Nagai, A., Kozawa, K., Kato, M., Nakajima, T., Mikami, T., Katsube, Y. \& Kimura, H. (2004). Genetic diversity of the dnaJ gene in the Mycobacterium avium complex. J Med Microbiol 53, 813-817.

Nei, M. \& Kumar, S. (2000). Molecular Evolution and Phylogenetics. New York: Oxford University Press.

Nhung, P. H., Shah, M. M., Ohkusu, K., Noda, M., Hata, H., Sun, X. S., lihara, H., Goto, K., Masaki, T. \& other authors (2007). The dnaJ gene as a novel phylogenetic marker for identification of Vibrio species. Syst Appl Microbiol 30, 309-315.

Olsen, G. J. \& Woese, C. R. (1993). Ribosomal RNA: a key to phylogeny. FASEB J 7, 113-123.
Peden, J. F. (2000). Analysis of Codon Usage. Nottingham: University of Nottingham.

Rivas, R., Velázquez, E., Valverde, A., Mateos, P. F. \& MartínezMolina, E. (2001). A two primers random amplified polymorphic DNA procedure to obtain polymerase chain reaction fingerprints of bacterial species. Electrophoresis 22, 1086-1089.

Rivas, R., Laranjo, M., Mateos, P. F., Oliveira, S., Martinez-Molina, E. \& Velázquez, E. (2007). Strains of Mesorhizobium amorphae and Mesorhizobium tianshanense, carrying symbiotic genes of common chickpea endosymbiotic species, constitute a novel biovar (ciceri) capable of nodulating Cicer arietinum. Lett Appl Microbiol 44, 412418.

Saito, H. \& Uchida, H. (1978). Organization and expression of DnaJ and DnaK genes of Escherichia coli K12. Mol Gen Genet 164, 1-8.

Saitou, N. \& Nei, M. (1987). The neighbor-joining method: a new method for reconstructing phylogenetic trees. Mol Biol Evol 4, 406425.

Seshadri, R., Joseph, S. W., Chopra, A. K., Sha, J., Shaw, J., Graf, J., Haft, D., Wu, M., Ren, Q. \& other authors (2006). Genome sequence of Aeromonas hydrophila ATCC $7966^{\mathrm{T}}$ : jack of all trades. J Bacteriol 188, $8272-8282$.

Shah, M. M., lihara, H., Noda, M., Song, S. X., Nhung, P. H., Ohkusu, K., Kawamura, Y. \& Ezaki, T. (2007). dnaJ gene sequence-based assay for species identification and phylogenetic grouping in the genus Staphylococcus. Int J Syst Evol Microbiol 57, 25-30.

Sharp, P. M., Bailes, E., Grocock, R. J., Peden, J. F. \& Sockett, R. E. (2005). Variation in the strength of selected codon usage bias among bacteria. Nucleic Acids Res 33, 1141-1153.

Siegenthaler, R. K., Grimshaw, J. P. A. \& Christen, P. (2004). Immediate response of the DnaK molecular chaperone system to heat shock. FEBS Lett 562, 105-110.

Somasegaran, P. \& Hoben, H. J. (1994). Handbook for Rhizobia. New York: Springer.

Stackebrandt, E., Murray, R. G. E. \& Trüper, H. G. (1988). Proteobacteria classis nov., a name for the phylogenetic taxon that includes the "purple bacteria and their relatives". Int J Syst Bacteriol 38, 321-325.

Stepkowski, T., Czaplinska, M., Miedzinska, K. \& Moulin, L. (2003). The variable part of the $d n a K$ gene as an alternative marker for phylogenetic studies of rhizobia and related alpha Proteobacteria. Syst Appl Microbiol 26, 483-494.

Swofford, D. L. (2003). PAUP ${ }^{*}$ : Phylogenetic analysis using parsimony (* and other methods). Sunderland, MA: Sinauer Associates.

Tamura, K., Dudley, J., Nei, M. \& Kumar, S. (2007). MEGA4: molecular evolutionary genetics analysis (MEGA) software version 4.0. Mol Biol Evol 24, 1596-1599.

Thompson, J. D., Higgins, D. G. \& Gibson, T. J. (1994). CLUSTAL W: improving the sensitivity of progressive multiple sequence alignment through sequence weighting, position-specific gap penalties and weight matrix choice. Nucleic Acids Res 22, 4673-4680.

Thompson, C. C., Thompson, F. L., Vandemeulebroecke, K., Hoste, B., Dawyndt, P. \& Swings, J. (2004). Use of recA as an alternative phylogenetic marker in the family Vibrionaceae. Int J Syst Evol Microbiol 54, 919-924.

Turner, S. L. \& Young, J. P. (2000). The glutamine synthetases of rhizobia: phylogenetics and evolutionary implications. Mol Biol Evol 17, 309-319.

Ventura, M., Canchaya, C., Bernini, V., Del Casale, A., Dellaglio, F., Neviani, E., Fitzgerald, G. F. \& van Sinderen, D. (2005). Genetic characterization of the Bifidobacterium breve UCC 2003 hrcA locus. Appl Environ Microbiol 71, 8998-9007. 
Vinuesa, P., Silva, C., Lorite, M. J., Izaguirre-Mayoral, M. L., Bedmar, E. J. \& Martínez-Romero, E. (2005). Molecular systematics of rhizobia based on maximum likelihood and Bayesian phylogenies inferred from rrs, $a t p D, \operatorname{rec} A$ and nifH sequences, and their use in the classification of Sesbania microsymbionts from Venezuelan wetlands. Syst Appl Microbiol 28, 702-716.

Vitorino, L., Chelo, I. M., Bacellar, F. \& Zé-Zé, L. (2007). Rickettsiae phylogeny: a multigenic approach. Microbiology 153, 160-168.

Xiao, X., Wang, P., Zeng, X., Bartlett, D. H. \& Wang, F. P. (2007). Shewanella psychrophila sp nov and Shewanella piezotolerans sp nov., isolated from west Pacific deep-sea sediment. Int J Syst Evol Microbiol $57,60-65$.
Yamamoto, S. \& Harayama, S. (1995). PCR amplification and direct sequencing of $g y r B$ genes with universal primers and their application to the detection and taxonomic analysis of Pseudomonas putida strains. Appl Environ Microbiol 61, 1104-1109.

Yamamoto, S., Kasai, H., Arnold, D. L., Jackson, R. W., Vivian, A. \& Harayama, S. (2000). Phylogeny of the genus Pseudomonas: intrageneric structure reconstructed from the nucleotide sequences of gyrB and rpoD genes. Microbiology 146, 2385-2394.

Young, J. P., Crossman, L., Johnston, A., Thomson, N. R., Ghazoui, Z. F., Hull, K. H., Wexler, M., Curson, A. R., Todd, J. D. \& other authors (2006).

The genome of Rhizobium leguminosarum has recognizable core and accessory components. Genome Biol 7, R34. 